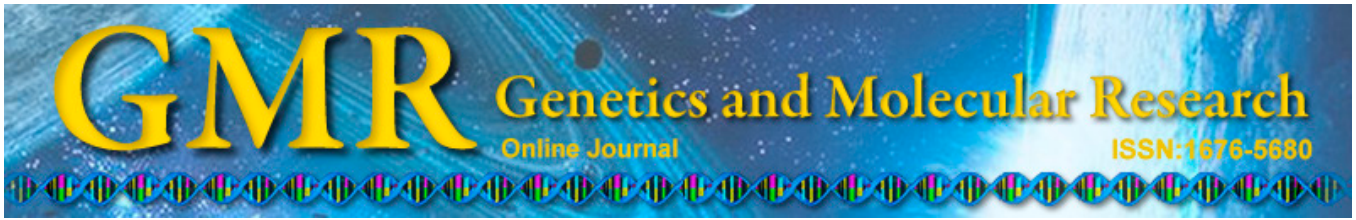

\title{
Effects of microwave ablation or its combination with whole-body chemotherapy on serum vascular endothelial growth factor levels in patients with stage IIIB/IV NSCLC
}

\author{
Y.H. Sun ${ }^{1,2}$, P.Y. Song ${ }^{1,2}$, Y. Guo ${ }^{1,2}$, M. Pang ${ }^{1,2}$, W.-N. He ${ }^{1,2}$, W.H. Zhang ${ }^{1,2}$ and \\ L.J. Sheng ${ }^{1,2}$ \\ ${ }^{1}$ Affiliated Hospital of ShanDong Academy of Medical Sciences, Shandong, \\ China \\ ${ }^{2}$ ShanDong Tumor Hospital, The East Campus, Shandong, China \\ Corresponding author: L.J. Sheng \\ E-mail: sunyahong_syh@163.com \\ Genet. Mol. Res. 14 (3): 10015-10025 (2015) \\ Received October 31, 2014 \\ Accepted March 18, 2015 \\ Published August 21, 2015 \\ DOI http://dx.doi.org/10.4238/2015.August.21.8
}

\begin{abstract}
We aimed to analyze the changes of serum vascular endothelial growth factor (VEGF) before and after microwave ablation (MWA) in patients with stage IIIB-IV non-small cell lung cancer (NSCLC), and to observe the effects of MWA combined with chemotherapy on short-term therapeutic efficacy and long-term survival. Concentrations of serum VEGF in 20 healthy subjects were considered as controls. Changes of serum VEGF were detected by ELISA before and after MWA ( 1 and 7 days after treatment). Seven days after MWA, patients were divided into a combination chemotherapy group of 22 subjects and a MWA alone group of 18 subjects. Serum VEGF was measured 1 month after MWA and 4 cycles after chemotherapy (3 months) to evaluate term effects; and 1- and 2-year survival rates. Serum VEGF concentrations declined sharply 1 day after MWA, and were significantly different from the levels before treatment. Subsequently,
\end{abstract}


VEGF rebounded 7 days after ablation, higher than that before treatment. At 1 and 3 months, serum VEGF levels in both treatment groups were remarkably lower than that before treatment; efficiency, or for the 1-year survival rate. However, the 2-year survival rates were significantly different between the two groups. We demonstrated that after MWA, the serum VEGF concentration undergoes a process of increasing, which might promote metastasis and recurrence of NSCLC. MWA combined with whole-body chemotherapy appears to be an effective method to increase the disease control rate, reduce the probability of metastasis and recurrence, and thus improve long-term survival.

Key words: Microwave ablation; Non-small cell lung cancer; Vascular endothelial growth factor

\section{INTRODUCTION}

The majority of patients who have been diagnosed with stage IIIB-IV non-small cell lung cancer (NSCLC) are inoperable and they gain little benefit from conventional chemotherapy and radiotherapy. Consequently, many novel local therapies have emerged, including local ablation therapy and stereotactic radiotherapy (Hadziahmetovic et al., 2010). In recent years, with the development of modern imaging techniques, image-guided minimally invasive tumor ablation has rapidly developed worldwide and has become increasingly important in comprehensive clinical tumor treatments. VEGF plays a crucial role in tumor angiogenesis, growth, recurrence, invasion, and metastasis; thus, its expression in the serum of patients with lung cancer and the clinical significance thereof has drawn much concern. As a local minimally invasive therapy, microwave ablation (MWA) effects changes in serum VEGF in patients before and after treatment. When combined with whole-body chemotherapy, this treatment has been shown to contribute to controlling tumor recurrence and metastasis, a process which worthy of additional research. However, the tumor microenvironment and its role in recurrence and metastasis have not yet attracted the attention of clinicians. There are few reports on the changes of VEGF related to MWA and on the survival benefit of its combination with chemotherapy. The present study aimed to detect the changes of serum VEGF in patients with NSCLC before and after MWA, analyze their clinical significance, examine the effect of MWA in combination with chemotherapy on long-term survival rate, and preliminarily propose a reasonable basis for clinical treatment and prognosis using these methods.

\section{MATERIAL AND METHODS}

\section{General information}

A total of 40 patients with stage IIIB-IV NSCLC who received MWA in the Affiliated Hospital of Shandong Province Academy of Medical Sciences from April 2011 to February 2014 were enrolled in this study. The patients included 26 men and 14 women, aged from 32 to 74 years (mean age, 64.25 years) whose diagnoses had been confirmed by histopathology or cytology. There were 10 patients in stage IIIB and 30 in stage IV. Of the total, 26 patients 
were diagnosed with adenocarcinoma, while the remaining 14 were diagnosed with squamous cell carcinoma. Before MWA, 46 pulmonary nodules were detected by computed tomography (CT), with diameters ranging from 1.1-6.8 cm (average, $3.6 \mathrm{~cm}$ ); 20 nodules were $\geq 3 \mathrm{~cm}$ in diameter while 26 nodules were $<3 \mathrm{~cm}$. Following treatment with multi-cycle and multi-line chemotherapy, patients were evaluated for stability in lung lesions, and disappearance or stability in other metastatic sites; the survival of patients involved was estimated to be $\geq 3$ months; the Eastern cooperative oncology group (ECOG) score was $0-2$. We explained the risks and complications to patients and their families as part of the request for their permission for inclusion. After signing the informed consent forms of MWA, patients were enrolled in the study.

As controls, we enrolled 20 healthy individuals detected in the Physical Examination Center of the Affiliated Hospital of Shandong Province Academy of Medical Sciences. This group consisted of 12 men and 8 women aged 30-72 years (average 62.67 years).

\section{Methods}

\section{$M W A$}

In this study, we utilized the KY-2000 microwave multifunction therapeutic apparatus provided by Nanjing Kangyou Institute of Microwave Energy Application (Nanjing, China), of which the microwave frequency was $2450 \mathrm{MHz}$ and the output power was 0-100 W (continuously adjustable). Before the operation, the location, size, and number of lung tumors were confirmed by CT scanning in order to locate the puncture site on the body surface and to select the best puncture route. Generally, the site that was located on the maximum level of the tumor and was closest to the tumor center was chosen as the thoracic cutaneous puncture site. Great vessels and adjacent bones such as the rib and scapula were carefully avoided. The puncture direction (pointing directly to tumor center), entry angle, and depth were confirmed before the operation. During the operation, patients were told to hold their breath and a microwave antenna was pricked to the predetermined site. CT scanning was conducted again to confirm the puncture locus, and then the MWA instrument connected to a water-cooling device was used for treatment, with a microwave frequency of $2450 \mathrm{MHz}$, power of $60-70 \mathrm{~W}$, and duration of 6-10 min. The ablation range was $0.5-1.0 \mathrm{~cm}$ away from the edge of the tumor. Tumors with diameters $\leq 3 \mathrm{~cm}$ received application from one antenna; tumors with diameters $>3 \mathrm{~cm}$ received application from two antennas. When the ablation was completed, we told the patients to hold their breath and slowly pulled out the antenna to cauterize the tract. After the operation, CT scanning was immediately conducted to observe changes of the foci and to detect whether pneumothorax or hemothorax had occurred.

\section{Detection of serum VEGF}

Before treatment, 1 and 7 days, and 1 and 3 months (or after 4 cycles of chemotherapy) after treatment, $3 \mathrm{~mL}$ peripheral blood was collected and allowed to rest for $30 \mathrm{~min}$ at room temperature before being centrifuged for $10 \mathrm{~min}$ at $2000 \mathrm{r} / \mathrm{min}$ to obtain a supernatant, which was then stored at $-70^{\circ} \mathrm{C}$.

The concentration of serum VEGF in patients with lung cancer was detected with a VEGF ELISA kit (Shanghai Lanji Biological Science and Technology Co., Ltd., Shanghai, China), according to manufacturer instructions. The $\mathrm{OD}_{450}$ value was immediately (within 5 min) measured, then the OD value was taken for the vertical axis, and the standard density as 
the horizontal axis to draw the standard curve, by which we could find out the corresponding density according to the serum sample OD value.

\section{Post-operation research design}

After undergoing MWA, the 40 patients were divided into two groups: 22 in the combination group (MWA combined with chemotherapy), and 18 in the single-treatment group (MWA alone). Patients in the combination group received whole-body chemotherapy seven days after ablation. The optimum chemotherapy regimen was chosen on the basis of different histopathological types and ECOG scores. The chemotherapy regimens were as follows: PC regimen (Pemetrexed $0.5 \mathrm{~g} / \mathrm{m}^{2} i v$ drip d1; PDD $25 \mathrm{mg} / \mathrm{m}^{2} i v$ drip d1-3; q21d); GP regimen (GEM $1.0 \mathrm{~g} /$ $\mathrm{m}^{2} i v$ drip d1,8; PDD $25 \mathrm{mg} / \mathrm{m}^{2} i v$ drip d1-3, q21d); TP regimen (DOC $75 \mathrm{mg} / \mathrm{m}^{2} i v$ drip d1; PDD $25 \mathrm{mg} / \mathrm{m}^{2} i v$ drip d1-3, q21d); the above chemotherapy regimens continued for 4 cycles.

\section{Efficacy evaluation}

Local efficacy evaluation was classified as complete ablation and incomplete ablation, according to the Expert Consensus for Thermal Ablation of Primary and Metastatic Lung Tumors (2014 Edition) (Ye et al., 2014). Endpoint efficacy evaluation of the combination and single-treatment groups referred to the response evaluation criteria in solid tumors, RECIST (2000), with classification as complete response (CR), partial response (PR), stable disease (SD), and progressive disease (PD); the disease control rate (DCR) was equal to $\mathrm{CR}+\mathrm{PR}+\mathrm{SD}$; patients were followed up for 1- and 2-year survival rates.

\section{Adverse effects}

Adverse effects of chemotherapy drugs were assessed in accordance with the World Health Organization (WHO) Anticancer Drugs Toxic Reaction Criteria, which were divided into degrees 0-IV.

\section{Statistical analysis}

All analyses were performed using the SPSS16.0 software (SPSS, Chicago, IL, USA). Results of measurement data are reported as means \pm standard deviation. Efficacy, disease control rates and survival rates were compared with a $\chi^{2}$ test between two groups. An independent samples $t$-test was applied for assessing the differences between two groups, while a paired samples $t$-test was used to analyze the differences between two groups before and after treatment. $\mathrm{P}<0.05$ was considered to be statistically significant.

\section{RESULTS}

\section{Local effects on tumors}

We examined 40 patients by enhanced CT scanning 1 month after MWA, and they all had reached a status of complete ablation without residual cancer and intrapulmonary recurrence. 


\section{Changes in VEGF concentration}

\section{Normal control vs NSCLC before and after MWA}

The average concentration of serum VEGF of the NSCLC group was $(259.79 \pm 57.34)$ $\mathrm{pg} / \mathrm{mL}$ (range 153.9-426.7), which was significantly higher $(\mathrm{P}<0.05)$ than that of the normal group [average $(98.24 \pm 26.99) \mathrm{pg} / \mathrm{mL}$, range (56.8-159.6)]. On the day after ablation, the mean concentration of serum VEGF of the 40 patients with NSCLC was $(108.61 \pm 33.26) \mathrm{pg} /$ $\mathrm{mL}$, and at 7 days after ablation, the mean rose up to $(327.62 \pm 57.71) \mathrm{pg} / \mathrm{mL}$. Compared with the mean concentration before ablation by paired-sample $t$-test, both values were considered statistically different $(\mathrm{P}>0.05$, Figure 1).

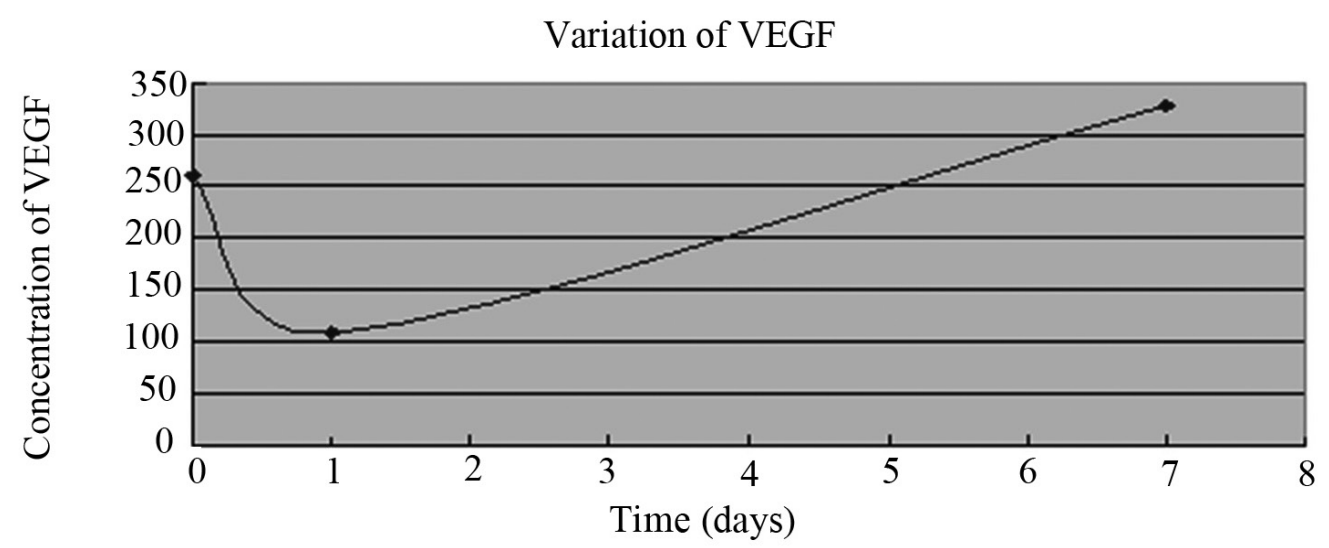

Figure 1. Time variation of the serum VEGF concentrations of 40 patients with NSCLC before and after MWA. VEGF, serum vascular endothelial growth factor; NSCLC, non-small cell lung cancer: MWA, microwave ablation.

As seen from Figure 1, the concentration of serum VEGF declined sharply the first day after treatment and was significantly different from that before treatment. At the 7th day after MWA, the level of VEGF increased and was remarkably higher than that before treatment.

\section{Combination vs single-treatment groups}

By independent-sample $t$-test, no significant difference was found in the concentration of VEGF between the combination and single-treatment groups before chemotherapy (the 7th day after MWA); following comparison by the paired-sample $t$-test, the serum VEGF levels in the 22 patients of the combination group at the 7th day after MWA (before chemotherapy) were dramatically different $(\mathrm{P}<0.05)$ from results detected one month after ablation and from those after 4 cycles of chemotherapy, respectively. Using the same statistical test, the serum VEGF levels in the 18 patients in the single-treatment group at the 7th day after MWA were found to be obviously different $(\mathrm{P}<0.05)$ from the values assessed at one and three months after MWA, respectively (Table 1, Figure 2).

As seen from Table 1 and Figure 2, the serum concentrations of VEGF in the 40 patients with NSCLC declined one month after MWA, and were significantly lower than those before treatment; the value remained low three months after treatment. 
Table 1. Variation in VEGF levels between the combination and single-treatment groups before and after chemotherapy $(\mathrm{pg} / \mathrm{mL})$.

\begin{tabular}{|c|c|c|c|}
\hline Groups & $\begin{array}{l}\text { VEGF before } \\
\text { chemotherapy }\end{array}$ & $\begin{array}{l}\text { VEGF } 1 \text { month } \\
\text { after } \text { MWA }^{2}\end{array}$ & $\begin{array}{l}\text { VEGF after } 4 \text { cycles of chemotherapy } \\
\text { or } 3 \text { months after } \text { MWA }^{3}\end{array}$ \\
\hline Combination & $327.62 \pm 57.71$ & $187.27 \pm 21.22$ & $209.79 \pm 57.34$ \\
\hline Single-treatment & $323.84 \pm 60.15$ & $200.67 \pm 51.15$ & $216.62 \pm 69.84$ \\
\hline
\end{tabular}

${ }^{1}$ By independent-sample $t$-test, no statistical difference was found in the levels of serum VEGF between the combination and single-treatment groups before chemotherapy; ${ }^{2}$ by independent-sample $t$-test, no statistical difference was found in the levels of serum VEGF between combination group and single-treatment group 1 month after MWA; ${ }^{3}$ By independent-sample $t$-test, no statistical difference was found between the serum VEGF levels of the combination group (detected after 4 cycles) and that of the single-treatment group (detected 3 months after MWA). VEGF = serum vascular endothelial growth factor; MWA = microwave ablation.

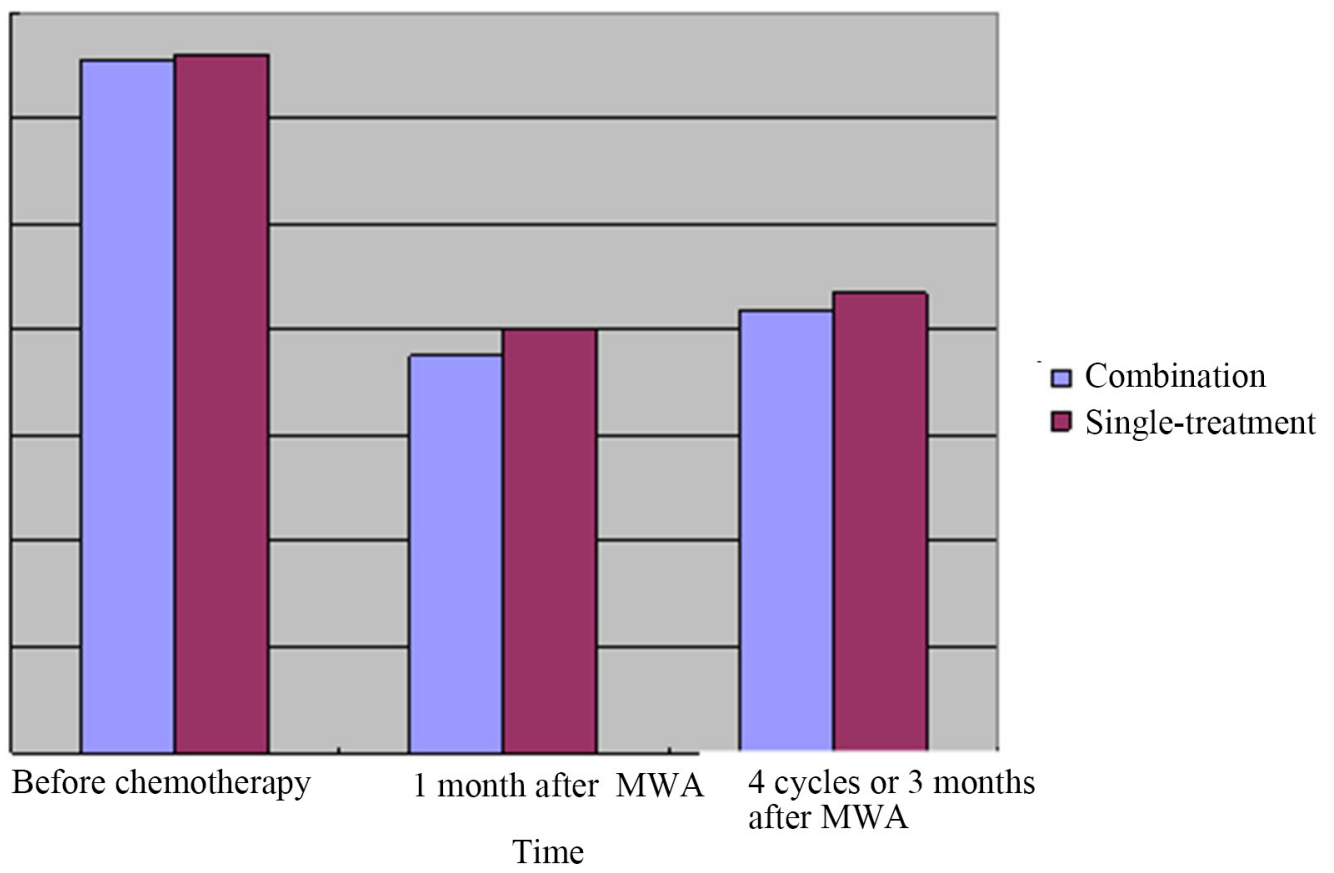

Figure 2. Changes in the concentrations of VEGF in the combination and single-treatment groups before and after chemotherapy $(\mathrm{pg} / \mathrm{mL})$.

\section{Short-term efficacy}

None of the patients in the two groups reached a status of CR. In the combination group, 2 reached $\mathrm{PR}, 15$ acquired $\mathrm{SD}$, and 5 presented as $\mathrm{PD}$, with a response rate (RR) of $9.1 \%(2 / 22)$ and a disease control rate (DCR) of $77.3 \%(17 / 22)$. In the single-treatment group, there was one patient with PR, seven with $\mathrm{SD}$, and ten with $\mathrm{PD}$, with $\mathrm{RR}=5.6 \%(1 / 18)$ and $\mathrm{DCR}=44.4 \%(8 / 18)$. No statistical difference $\left(\chi^{2}=0.33, \mathrm{P}>0.05\right)$ was found when comparing the RRs between the two groups; in contrast, the DCRs were considered significantly different from one another following comparison of the two groups $\left(\chi^{2}=4.55, \mathrm{P}<0.05\right)$. 


\section{Long-term survival rate}

Follow-up (6-35 months) and analysis of the survival rates of two groups showed that a total of three patients (one in the combination group and two in the single-treatment group) were lost to follow-up (considered as death). The 1- and 2-year survival rates of the combination group were 77.3 and $59.1 \%$, respectively. The 1- and 2-year survival rates of the singletreatment group were 50 and $27.8 \%$, respectively. There was no statistical significance $(\mathrm{P}>$ 0.05 ) between the 1-year survival rates of the two groups, whereas the 2-year survival rates were significantly different between the groups $(\mathrm{P}<0.05)$.

\section{Adverse effects}

Complications associated with MWA included pneumothorax, infection, pleural reaction and hemothorax, and these were observed in 13 (32.5\%), 7 (17.5\%), $1(2.5 \%)$, and $4(10 \%)$ patients, respectively. Of these, four patients with pneumothorax required chest tube intervention and the tubes were removed within seven days; other complications required no special treatment.

Adverse effects associated with MWA combined with chemotherapy varied between chemotherapy regimens. The main adverse effects that resulted from the PC scheme were hypodynamia and skin pigmentation, while those from the GP and TP schemes were gastrointestinal reactions and myelosuppression. Following analysis by the $\chi^{2}$ test, the differences in gastrointestinal reactions between the combination and single-treatment groups were shown to be statistically significant $(\mathrm{P}<0.05)$; furthermore, there was also a remarkable difference $(\mathrm{P}$ $<0.05)$ in myelosuppression between the two groups.

\section{DISCUSSION}

The formation of lung carcinoma is a very complicated process that is generally divided into two periods: the clonal growth period of tumor cells and the continued growth period promoted by subsequent angiogenesis. Tumorigenesis is closely related to changes in certain genes, alteration of the extracellular matrix and the cell fluid microenvironment, and positive and negative regulation of many cytokines such as fibroblast growth factor (FGF), transforming growth factor (TGF) (Nugent and Iozzo, 2005), platelet-derived growth factor (PDGF), and vascular endothelial growth factor (VEGF) (Petrova et al., 1999; Liu et al., 2003). Among these, VEGF is considered as the primary factor for stimulation of angiogenesis, and it participates in tumor vascular regeneration and in tumor growth and metastasis. Expression of VEGF has been shown to vary dramatically between different histological types of lung cancer, with a higher expressivity detected in adenocarcinoma but small amounts detected in squamous cell carcinoma. The expression of VEGF was shown to associate closely with lymph node and distant metastasis of lung cancer, the rates of these increase markedly in patients with positive VEGF expression (Bonnesen et al., 2009; Korpanty et al., 2010; Kajdaniuk et al., 2011; de Mello et al., 2012). Trapé et al. (2003) performed a quantitative study by ELISA, which showed that the serum VEGF levels of patients with lung cancer were remarkably higher than those of patients with benign disease and of healthy individuals $(\mathrm{P}<0.001)$. They therefore regarded the serum VEGF level as valuable for diagnosing lung cancer, though it does not correlate with either histological type or clinical stage. In contrast, Matsuyama et al. (2000) performed a study using the same methodology, but found that the serum VEGF levels were 
positively correlated with increasing clinical stages $(\mathrm{P}<0.0001)$, which suggested that VEGF levels can be applied to the speculation of disease prognosis. Most studies have demonstrated that expression of VEGF is in significant correlation with the prognosis of lung cancer and patients with high expression are given poor prognoses.

Generally speaking, patients with stage IIIB-IV NSCLC are unsuited for surgery; for these, the conventional standard treatment is a combination of radiotherapy and chemotherapy. The chemotherapy scheme is platinum-based doublet chemotherapy containing third -generation chemotherapeutic drugs such as pemetrexed, docetaxel, gemcitabine, and paclitaxel, among others. However, the efficiency of chemotherapy is merely $30-40 \%$, although it can be improved, though not much, by concurrent radiotherapy and chemotherapy when compared with that of single treatment. With the application of molecular-targeted drugs, the therapeutic efficacy in advanced NSCLC has been improved, but this is limited to patients with EGFR mutation. Most patients present with progressive disease during initial treatment, and even if their disease is under control at that point, it will ultimately progress. There are three main factors accounting for NSCLC treatment failure: the first is distant metastasis, the second is local loss of control, and the third is immune dysfunction. The first and the third factor are usually unchangeable, and thus most studies focusing on NSCLC treatment failure in recent years have been mainly concentrated on the second factor. Radiotherapies and percutaneous minimally invasive surgeries such as MWA are regarded as the most effective therapies for achieving local tumor control. Several studies have explored radiotherapy for primary tumor sites in patients with advanced lung cancer, and the results suggested that it was able to improve the local control rate, relieve the symptoms, and improve the survival rate. However, this benefit was mainly restricted to patients with good performance status and to those with metastases or single metastasis of limited volume (Nestle et al., 2000; Bezjak et al., 2002; Reinfuss et al., 2011; Hasselle et al., 2012). In addition, long radiotherapy intervals, long-term adverse effects, and the high costs confine the application of radiotherapy as well.

With nearly 20 years of development, MWA, as a minimally invasive treatment method, has achieved substantial therapeutic effects (Dong et al., 2003) in clinical practice, especially in the treatment of liver cancer. It not only leads to one-time complete inactivation of small hepatocellular carcinomas with diameters $\leq 5 \mathrm{~cm}$, but also results in activation of innate immunity (Wang et al., 2006). Because of the heat insulation effect produced by the pulmonary gas surrounding lung cancer, the thermal power can easily assemble to form a huge energy circle, which provides the opportunity for thermal ablation methodologies such as MWA to treat lung cancer (Gadaleta et al., 2004; Goldberg et al., 1995). MWA has presented new concepts for the minimally invasive treatment of lung cancer. It has been proven by numerous studies to be safe and effective, especially for patients insensitive to chemotherapy and for those with stable lung tumors. For these patients, MWA works by reducing the local tumor load, decreasing the chances of tumor recurrence and metastasis, and enhancing the innate immunity. Natharn et al. (2005) performed MWA guided by imaging on 45 patients with a total of 78 lung cancer lesions, of which the diameters ranged from 1-7 cm (average, $2.9 \mathrm{~cm}$ ). That study found that the ablation diameter could reach $4.8 \mathrm{~cm}$ and that MWA was able to ablate lesions $<3.0 \mathrm{~cm}$ completely with a single electrode; the effective rate was $97.6 \%(40 / 41)$, and the recurrence rate was $26.8 \%$ (11/41); among 11 patients with recurrence, 8 had lung metastases. In the study of $\mathrm{He}$ et al. (2007) on MWA treatment for peripheral lung cancer, 17 patients with a total of 23 tumor nodules were followed up for 6 to 47 months (mean 23 months); therein, the lesions were shown to have shrunk to different degrees with disappear- 
ance or reduction of the internal flow signal after treatment; enhanced CT revealed that 13 tumor nodules had no enhancement, while the other seven received partial enhancement; after treatment, tumors reached complete necrosis in the ablation area in four patients; one month after MWA, clinical symptoms disappeared in nine patients, and eased in nine. Carrafiello et al. (2010) recommended MWA to replace other ablations and to be the first choice for some patients. The further analysis of the effects on growth factors that promote tumor growth, metastasis, and recurrence after microwave treatment would have a profound meaning for the further development of MWA. Consequently, the present study focused on the effects of MWA on peripheral VEGF, as well as the influence of its combination with chemotherapy on longterm survival in patients with NSCLC.

Our experimental results demonstrated that on the first day after microwave treatment, the concentration of serum VEGF declined sharply, and was significantly different from that before treatment. On the 7th day after treatment, the levels of VEGF rose again and were notably higher than those before treatment. At one month after treatment, the concentration of serum VEGF decreased to levels significantly lower than those before treatment. We considered that the reduction of VEGF on the first day after MWA might have resulted from radical cytoreduction of the local tumor, which declined the tumor burden and thus greatly reduced serum VEGF. Afterwards the process of lung regeneration began, with a concomitant increase of oxygen demand and VEGF levels owing to the demand for vascular regeneration, accounting for the increased VEGF levels one week after treatment. By the end of the first month, the general condition of the lung had stabilized, which accounted for the sharp reduction of serum VEGF to lower than the pretreatment level. Therefore, patients with lung cancer experienced an increase of serum VEGF after MWA. Yet, the increase of VEGF would likely accelerate the recurrence of lung cancer micrometastases if it already existed in the patient. Therefore, if treatments to control VEGF could be focused on this stage, tumor metastasis and recurrence would likely be reduced or delayed.

In addition, as a local treatment method, MWA has blind spots for destroying subclinical lesions and microcirculation metastases; these are common among local thermal ablation technologies. Furthermore, complete ablation cannot be achieved due to the anatomical position of the tumor lesion; yet recurrence often occurs around the lesion because of the incomplete ablation of the normal aerated lung tissue around the tumor, which is deemed as the main factor resulting in tumor progression. In contrast, as a whole-body treatment method, chemotherapy can achieve the goals in treating primary lesions, micrometastases, and subclinical lesions. Chemotherapy has advantages not available to local therapies, and thus can improve the effects of comprehensive treatments. Consequently, MWA combined with whole-body chemotherapy is feasible (Fahy and Jarnagin, 2006) in theory. To date, however, no studies have concentrated on MWA combined with chemotherapy to treat stage IIIB-IV NSCLC. Thus, we attempted to determine whether MWA combined with third-generation platinum-based doublet chemotherapy could improve the survival rates of patients with advanced NSCLC.

Based on the above reasons, we compared the short-term and long-term efficacy between the combination and the single-treatment groups on the 7th day after MWA. Experimental results suggested that no statistical differences could be found between the groups ( $9.1 \mathrm{vs}$ $5.6 \%, \mathrm{P}>0.05)$; however, comparison between the two groups in DCR showed a significant difference $\left(77.3\right.$ vs $\left.44.4 \%, \chi^{2}=4.55, \mathrm{P}<0.05\right)$; and whereas comparison between the two groups for the 1-year survival rates were not significantly significant (77.3 vs 50.0\%, P > 0.05 ), comparison between the two groups for the 2-year survival rate revealed a significant 
difference $(59.1$ v $27.8 \%, \mathrm{P}<0.05)$. We thought that the inverse situations in comparison of the efficacy and DCR between the two groups could be transformed into that from comparing the 1-year and 2-year survival rates. Currently, the third generation platinum-based doublet chemotherapy used for first-line treatment leads to a 1-year survival rate of $30-40 \%$ and a 2-year survival rate of $10-15 \%$. Our research showed that the 1- and 2-year survival rates of the two groups, and of the combination group in particular, were both higher than that assessed in studies focused on chemotherapy alone. From this, we can see that MWA combined with chemotherapy contributes to significant improvement of the long-term survival rate.

In treatment for end-stage NSCLC, MWA combined with chemotherapy takes its effect from mutual complementary and synergistic actions. The possible mechanisms include: 1) a reduction of tumor burden after local treatment and an increase of tumor proliferation that enhances patient sensitivity to chemotherapy; 2) MWA can completely destroy the tumor and achieve local cytoreduction; whereas chemotherapy can eliminate peripheral tumor cells and subclinical micrometastases to suppress recurrence and distant metastases in the whole body; they thus complement each other; 3) MWA is non-specific for tumor cells, whereas wholebody chemotherapy can target tumor cells in different stages; the combination of the two can achieve complementary advantages; and 4) MWA can enhance the native immune function and ameliorate to some degree the immune suppression caused by chemotherapy drugs.

\section{Conflicts of interest}

The authors declare no conflict of interest.

\section{REFERENCES}

Bezjak A, Dixon P, Brundage M, Tu D, et al. (2002). Randomized phase III trial of single versus fractionated thoracic radiation in the palliation of patients with lung cancer (NCIC CTG SC.15). Int. J. Radiat. Oncol. Biol. Phys. 54: 719-728.

Bonnesen B, Pappot H, Holmstav J and Skov BG (2009). Vascular endothelial growth factor A and vascular endothelial growth factor receptor 2 expression in non-small cell lung cancer patients: relation to prognosis. Lung Cancer 66: 314-318.

Carrafiello G, Mangini M, De Bernardi I, Fontana F, et al. (2010). Microwave ablation therapy for treating primary and secondary lung tumors: technical note. Radiol. Med. 115: 962-974.

de Mello RA, Costa BM, Reis RM and Hespanhol V (2012). Insights into angiogenesis in non-small cell lung cancer: molecular mechanisms, polymorphic genes, and targeted therapies. Recent. Pat. Anticancer. Drug. Discov. 7: 118131.

Dong B, Liang P, Yu X, Su L, et al. (2003). Percutaneous sonographically guided microwave coagulation therapy for hepatocellular carcinoma: results in 234 patients. AJR Am. J. Roentgenol. 180: 1547-1555.

Fahy BN and Jarnagin WR (2006). Evolving techniques in the treatment of liver colorectal metastases: role of laparoscopy, radiofrequency ablation, microwave coagulation, hepatic arterial chemotherapy, indications and contraindications for resection, role of transplantation, and timing of chemotherapy. Surg. Clin. North Am. 86: 1005-1022.

Gadaleta C, Mattioli V, Colucci G, Cramarossa A, et al. (2004). Radiofrequency ablation of 40 lung neoplasms: preliminary results. AJR Am. J. Roentgenol. 183: 361-368.

Goldberg SN, Gazelle GS, Compton CC, and McLoud TC (1995). Radiofrequency tissue ablation in the rabbit lung: efficacy and complications. Acad. Radiol. 2: 776-784.

Hadziahmetovic M, Loo BW, Timmerman RD, Mayr NA, et al. (2010). Stereotactic body radiation therapy (stereotactic ablative radiotherapy) for stage I non-small cell lung cancer-updates of radiobiology, techniques, and clinical outcomes. Discov. Med. 9: 411-417.

Hasselle MD, Haraf DJ, Rusthoven KE, Golden DW, et al. (2012). Hypofractionated image-guided radiation therapy for patients with limited volume metastatic non-small cell lung cancer. J. Thorac. Oncol. 7: 376-381. 
He W, Hu XD, Zuo DF, and Guo L (2007). Ultrasound-guided percutaneous microwave ablation of lung cancer. Chin. J. Min. Inv. Surg. 7: 406-408.

Kajdaniuk D, Marek B, Foltyn W and Kos-Kudła B (2011). Vascular endothelial growth factor (VEGF)-part 2. in endocrinology and oncology. Endocrinol. Pol. 62: 456-464.

Korpanty G, Smyth E, Sullivan LA, Brekken RA, et al. (2010). Antiangiogenic therapy in lung cancer: focus on vascular endothelial growth factor pathway. Exp. Biol. Med. (Maywood) 235: 3-9.

Liu J, Sun ZY and Cao L (2003). Effect of vascular endothelial growth factor on osteogenesis of bio-derived bone compounded with marrow stromal stem cells in vivo. Chin. J. Clin. Rehabil. 7: 1622-1623.

Matsuyama W, Hashiguchi T, Mizoguchi A, Iwami F, et al. (2000). Serum level of vascular endothelial growth factor dependent on the stage progression of lung cancer. Chest 118: 948-951.

Natharn A, Paul F, Lymm S, et al. (2005). Microwave ablation: organ specific approach to lung ablation. Cancer 104: 1271-1280.

Nestle U, Nieder C, Walter K, Abel U, et al. (2000). A palliative accelerated irradiation regimen for advanced non-smallcell lung cancer $v s$ conventionally fractionated $60 \mathrm{GY}$ : results of a randomized equivalence study. Int. J. Radiat. Oncol. Biol. Phys. 48: 95-103.

Nugent MA and Iozzo RV (2005). Fibroblast growth factor-2. Int. J. Biochem. Cell. Biol. 32: 115-120.

Petrova TV, Makinen T and Alitalo K (1999). Signaling via vascular endothelial growth factor receptors. Exp. Cell. Res. 253: $117-130$.

Reinfuss M, Mucha-Malecka A, Walasek T, Blecharz P, et al. (2011). Palliative thoracic radiotherapy in non-small cell lung cancer. An analysis of 1250 patients. Palliation of symptoms, tolerance and toxicity. Lung Cancer 71: 344-349.

Trapé J, Buxó J and de Olaguer JP (2003). Serum concentrations of vascular endothelial growth factor in advanced nonsmall cell lung cancer. Clin. Chem. 49: 523-525.

Wang ZL, Dong BW, Liang P, Yu XL, et al. (2006). Changes of serum vascular endothelial growth factor before and after microwave ablation or hepatic resection in patients with small hepatocellular carcinoma. China J. Mod. Med. 16: 3119-3121.

Ye X, Fan WJ and Minimally Invasive and Comprehensive Treatment of Lung Cancer Branch, Professional Committee of Minimally Invasive Treatment of Cancer, Chinese Anti-Cancer Association. (2014). Expert consensus for thermal ablation of primary and metastatic lung tumors. Zhongguo Fei Ai Za Zhi 17: 294-301. 